\title{
Original Alticle \\ Translation and validation of the Hindi version of chronic liver disease questionnaire (CLDQ) for the assessment of health related quality of life in patient with chronic liver disease in India
}

\author{
Jaya Benjamin ${ }^{1}$, Kalaivani Mani ${ }^{2}$, Anoop Saraya ${ }^{3}$, \\ Yogendra Kumar Joshi ${ }^{1}$
}

ABSTRACT

\begin{abstract}
${ }^{1}$ Department of Clinical Nutrition, Institute of Liver and Biliary Sciences, Vasant Kunj, New Delhi, ${ }^{2}$ Department of Biostatistics, All India Institute of Medical Sciences, New Delhi, ${ }^{3}$ Department of Gastroenterology \& Human Nutrition, All India Institute of Medical Sciences, New Delhi.
\end{abstract}

Correspondence: Yogendra Kumar Joshi Email:ykj2511@usa.net assess the validity of the Hindi CLDQ.
Background: Chronic liver disease questionnaire (CLDQ) is an established disease specific tool to assess the health related quality of life (HRQOL) in patients with CLD irrespective of the etiology and severity. The objective of the study was to develop the Hindi language version of CLDQ and to

Methods: CLDQ was adapted into Hindi language by following a standard method of forward and back-translation by two bilingual translators, revision by expert panel and formulation of a consensual version followed by pilot testing and appropriate modifications before final administration. Hindi CLDQ along with short form 36 (SF-36) were administered in 494 patients with chronic liver disease (CLD) and 103 healthy controls (HC). To assess reproducibility, Hindi CLDQ was re-administered in a subsample of 20 patients. Internal consistency was assessed by Cronbach's alpha coefficient. Structural validity was assessed by exploratory factor analysis and construct validity was assessed with help of correlation of SF-36 scores and CLDQ pattern across disease severity groups.

Results: The Cronbach's alpha coefficient was 0.93 and intra class coefficient correlation was 0.88 for the average CLDQ score, suggestive of good reliability. An inverse relationship of the average CLDQ score with the disease severity (CLDQ score of Child A vs. B vs. C $=5.2 \pm 1.10$ vs. $4.73 \pm 1.24$ vs. $4.15 \pm 1.19$ respectively; $\mathrm{p}<0.001)$ and good correlation with SF-36 scores (average CLDQ score with PCS \& MCS; $r=0.63 \&$ $0.55 ; \mathrm{p}<0.001)$ was suggestive of good validity of Hindi CLDQ. The factor analysis identified 6 domains accounting for $62 \%$ of variability. The derived cut-off of 6 for the average CLDQ had the AUC of 0.826 with a sensitivity of 82.6 and a specificity of 76.7 .

Conclusion: Hindi version of CLDQ is found to be a reliable and a valid tool comparable to the original English version. The HRQOL of patients with CLD is lower than healthy controls and HRQOL decreases with increase in disease severity.

KEYWORDS: CLDQ; HRQOL; hindi version. 


\section{Introduction}

Chronic liver disease (CLD), one of the leading health concerns in India has a tortuous clinical course and high mortality risk. Disease related severe disease complications along with endoscopic procedures, side effects of drug therapy and associated co-morbidities further add insult to injury. Additionally non-specific symptoms, major nutritional concerns and emotional issues profoundly affect the general well being of these patients. Since the advances in medical therapy have significantly prolonged the overall survival, alleviation of symptoms and improvement in the overall quality of life is of paramount importance and a desirable endpoint of medical therapy. Hence along with longevity, the quality of life has gained much importance.

Health related quality of life (HRQOL) is a multidimensional concept that is a quantitative estimation of patient's self assessment of the physical, functional, social and psychological dimensions of life. ${ }^{1}$ HRQOL is now considered as an objective tool for measuring the outcome of medical interventions in various trials and more recently its importance has been appreciated in the long term management after liver transplantation. ${ }^{2} \mathrm{~A}$ wide variety of tools have been described for the assessment of HRQOL. These tools can broadly be classified as generic or disease specific, usually the two types complement each other. For CLD, chronic liver disease questionnaire (CLDQ) is a widely acknowledged tool which is wholesome yet concise, easy to execute and applicable to all the patients irrespective of the etiology and severity of the disease.

The original English version of CLDQ by Younossi et $\mathrm{al}^{3}$ is a popular instrument whose validity, reliability and responsiveness has been well established. ${ }^{4-6}$ It has been translated and validated into various languages all over the world including both European (German, ${ }^{7}$ Spanish, ${ }^{8}$ Italian, ${ }^{9}$ Swedish, ${ }^{10}$ Greek $^{11}$ ) and Asian (Persian, ${ }^{12}$ Thai,${ }^{13}$ Japanese, ${ }^{14}$ Chinese, ${ }^{15}$ Bengali ${ }^{16}$ and Srilankan ${ }^{17}$ ) languages. Such a cross cultural adaptation enables global comparisons of HRQOL data and also helps in designing international projects. There was a need for a Hindi version of the CLDQ that could retain the conceptual and functional aspects of the original English version and yet express linguistic and cultural differences in the issues related to the HRQOL. Hence this study was planned to translate \& validate the Hindi version of CLDQ.

\section{Methods and Materials}

\section{Chronic Liver Disease Questionnaire}

Chronic liver disease questionnaire (CLDQ) is a disease specific tool developed by Younossiet al. ${ }^{3}$ for the assessment of the HRQOL in patients with CLD. It contains 29 questions summarized into 6 domains namely abdominal symptoms, fatigue, systemic symptoms, activity, emotional functioning, and worry. Each item is ranked on a 7 point Likert scale ranging from 1 (all the time, i.e. indicating the worst HRQOL) to 7 (none of the time, i.e. indicating the best HRQOL) for a period of previous two weeks. Six subscale scores and the overall CLDQ score are the means of the items and all the domains respectively.

\section{Short Form-36}

The validated Hindi version of original Short Form 36 $(\mathrm{SF}-36)^{18}$ a generic HRQOL tool was purchased from the Quality Metric health Outcomes Solutions ${ }^{19}$ Inc. USA, and also administered on the patients.

\section{Adaptation of CLDQ to Hindi Language}

A standard method ${ }^{20,21}$ was used for the adaptation of the original English version of CLDQ into Hindi language. Two bilingual translators, adept at both Hindi and English and whose mother tongue was Hindi, performed independent forward translations. While translating the conceptual equivalence was given importance over linguistic equivalence. An expert panel comprising of hepatologists and research scientists undertook a joint revision to form a consensual version and also to test the equivalence between the consensual Hindi translation and the original English version. 
The questionnaire was initially administered on 10 stable patients with CLD to identify any difficulty they experienced while filling out the questionnaire. There were problems in differentiating between 'felt sleepy' and 'felt drowsy' in the fatigue domain and in understanding 'shortness of breath' in the systemic domain; these problems were resolved by discussion with the experts. In the emotion domain the English word 'mood' was retained for a better understanding.Attention was given to the common parlance of the patients rather than verbatim Hindi translation. Two back translations of the Hindi version were made and when contrasted with the original questionnaire, were found comparable.

\section{Study Subjects}

All consecutive patients with chronic liver disease attending the Liver clinic and Gastroenterology OPD of the department of Gastroenterology and Human Nutrition, AIIMS, from September 2009 to November 2012 were enrolled in this study as per the inclusion and exclusion criteria.

The diagnosis of CLD and the etiology was based on the standard histological, clinical, biochemical and radiological criteria. Age and gender matched individuals who were the patient's attendants or staff members (free from any known physical or mental illness requiring treatment) served as healthy controls for the HRQOL assessment.

\section{Inclusion and Exclusion criteria}

All the patients with CLD aged between 15 to 80 years of either gender were enrolled consecutively. However, patients with overt hepatic encephalopathy, active medical co-morbidities, malignancies, cognitive difficulties or psychiatric disorders were excluded from the study. All the patients underwent a detailed clinical evaluation and information was collected regarding the socio-demographic profile, duration of symptoms, etiology, severity of disease, routine biochemical and hematological investigations, upper GI endoscopic findings and radiological investigations. The severity of the disease was assessed using the Child-Pugh Score.

\section{Administration of $C L D Q$}

Patients and healthy controls were asked to complete the CLDQ and SF36 questionnaires after explaining the nature of the study and giving them a broad idea about the questionnaires. These questionnaires were filled during the regular outpatient visits. Literate candidates filled the questionnaires on their own while trained research personnel helped interviewing the illiterate candidates, checked the completeness of the answered questionnaires and gave instructions when needed to invite the candidate to fill in the missing items.In a subsample of 20 patients the Hindi CLDQ was re-administered two weeks later to assess the reproducibility.

\section{Assessment of Reliability}

Reliability of Hindi CLDQ (which refers to the precision of a measure) was assessed by two methods 1) The internal consistency defined as precision based on homogeneity (i.e. the degree to which the set of items or questions measures a single unidimensional latent construct or domain) was evaluated using the Cronbach's alpha coefficient and 2) reproducibility or consistency was assessed by the test-retest analysis expressed as intraclass correlation coefficient (ICC) ranging from 0 (no agreement) to 1 (perfect agreement).

\section{Assessment of Validity}

Validity (which refers to the instrument's ability to truly measure what it intends to measure) was assessed in two ways-1) Structural Validity: Exploratory factor analysis with varimax rotation was carried out to assess whether placement of items into domains of the Hindi CLDQ followed the distribution of the original English questionnaire. Factors were selected for a maximum cumulative variance explained with an Eigenvalue $\geq 1$ ) Construct Validity:The extent to which the scores 
demonstrate expected logical relations with other HRQOL and clinical measures was assessed by estimating the difference in the CLDQ scores of patients with different disease severity and also by comparing the CLDQ sores with the SF-36 scores.

\section{Statistical Analysis}

Analysis was carried out using Stata 11.0 (Texas, USA). Data were presented as number (percentage) and mean $\pm \mathrm{SD}$. The mean difference in means of HROQL scores between patients and controls were compared using Student's t test for independent samples and across child status within patients was compared using one-way ANOVA followed by Bonferroni correction. Correlation between CLDQ and SF-36 was calculated using Pearson's correlation coefficient. The diagnostic accuracy of CLDQ for assessing HRQOL in patients against controls was analyzed using receiver operating characteristic (ROC) curve and results were presented as AUC (95\% CI). The p-value less than 0.05 was considered as statistically significant.

\section{Results}

A total of 494 patients with CLD(age: $42.3 \pm 12.2(18-60)$ years; Males: $85.2 \%$ ) and 103 controls (age: $40.3 \pm 12.0$ (17-74 yrs); males: $82.5 \%$ ) were included in the study. The clinical characteristics of the patients are shown in Table 1.

The most common etiology of CLD was alcohol followed by viral hepatitis. Nearly $66 \%$ of the patients were decompensated, with an average duration of disease of 24 months. Most of the patients had a minimum of higher secondary to intermediate level of education (64.4\%), were clerks-shopowners-farmers by profession and belonged to the lower level $(85.9 \%)$ of socioeconomic strata. Majority of the patients $(88.1 \%)$ were married. A small subset of 20 patients that constituted the reproducibility cohort comprised of 16 males and 4 females with a predominant etiology of alcoholism followed by viral. Their mean age was $40 \pm 23$ years. Most of the patients were in the Child class B.
Table 1: Clinical characteristics of patients with CLD

\begin{tabular}{l|l} 
Variables & Patients (n=494) \\
Age (years) & $42.3 \pm 12.26$ \\
\hline Gender (Male(\%):Female(\%)) & $421(85.2): 73(14.8)$ \\
\hline Etiology & \\
\cline { 2 - 2 } $\begin{array}{l}\text { Alcoholic } \\
\text { Viral }\end{array}$ & $182(36.8)$ \\
\cline { 2 - 2 } Others & $164(33.2)$ \\
\cline { 2 - 2 } Child Status & $148(30.0)$ \\
$\begin{array}{l}\text { B } \\
\text { C }\end{array}$ & $164(33.2)$ \\
\hline Duration of disease (months) & $198(40.0)$ \\
\cline { 2 - 2 } Portal hypertension & $132(25.7)$ \\
\hline Varices & $465(1-180)$ \\
\hline Portal hypertensive gastropathy & $231(47.9)$ \\
\hline
\end{tabular}

Data are expressed as mean $\pm S D$ and number (percentage).

Reliability and Reproducibility of Hindi version of chronic liver disease questionnaire (CLDQ)

The distribution characteristics and the reliability of the CLDQ scores are shown in Table 2. Cronbach's alpha, which is a measure of internal consistency ranged from 0.62 in the systemic domain to 0.85 in the emotional domain. The alpha value exceeded 0.70 in all domains except for systemic symptoms. The overall Cronbach's alpha was excellent i.e. 0.93. The measure of reproducibility of the Hindi CLDQ assessed in a subsample of 20 patients was good, ranging from 0.74 in the activity domain to 0.92 in the abdominal symptoms domain and the Inter-class consistency of the test retest CLDQ score was excellent at 0.88 . SF-36 also showed good reliability as the Crohbach's alpha of all the domains of SF36 questionnaire were more than 0.70 i.e. Physical Functioning (PF: 0.92), Role Physical (RP:0.89), Body Pain(BP:0.84), Vitality (VT:0.74), Social Functioning (SF: 0.77), Role Emotional (RE :0.90)and Mental Health (MH:0.75) except for the domain of general health (GH:0.65). 
Table 2: Reliability of Hindi CLDQ in patients with CLD

\begin{tabular}{l|l|l|l|l} 
CLDQ domain & $\begin{array}{l}\text { Mean Score } \\
\text { Mean } \pm \text { SD }\end{array}$ & $\begin{array}{l}\text { Cronbach's alpha } \\
(\mathbf{n}=\mathbf{4 9 4})\end{array}$ & $\begin{array}{l}\text { Test-retest reliability } \\
(\mathbf{n = 2 0}) \\
\text { Correlation } \\
\text { coeffecient }\end{array}$ & P value \\
\hline 1. Abdominal symptoms & $4.91 \pm 1.64$ & 0.70 & 0.92 & 0.0001 \\
\hline 2. Fatigue & $4.24 \pm 1.54$ & 0.81 & 0.80 & 0.0001 \\
\hline 3. Systemic symptoms & $5.10 \pm 1.25$ & 0.62 & 0.76 & 0.0001 \\
\hline 4. Activity & $4.66 \pm 1.86$ & 0.77 & 0.74 & 0.0001 \\
\hline 5. Emotional function & $4.72 \pm 1.40$ & 0.85 & 0.76 & 0.0001 \\
\hline 6. Worry & $4.70 \pm 1.52$ & 0.81 & 0.90 & 0.0001 \\
\hline Average CLDQ score & $4.72 \pm 1.25$ & 0.93 & 0.88 & 0.0001
\end{tabular}

Validity of Hindi version of chronic liver disease questionnaire $(C L D Q)$

\section{Construct Validity}

As shown in Table 3 the individual domain \& overall CLDQ scores of the patients were significantly lower than that of the controls. Except for the domains of abdominal symptoms, fatigue, systemic symptoms between patients with Child status A and B and for worry between patients with Child status $B$ and $C$ there was a significant difference in the individual domain and overall scores between patients with different disease severity. An average decrease of 0.50 points was seen in the overall CLDQ score with increasing disease severity in our patients with CLD. There was no significant difference between patients with Child A and B for all the domain scores and component summary scores of SF-36. The scores of Role emotional (RE) and MCS were comparable irrespective of the disease severity.

\section{Convergent Validity}

The correlation values of all the 6 domains and average CLDQ score with the 8 domains and summary scores of SF-36 is shown in Table 4. A significant $(p<0.001)$ correlation was seen between both the questionnaires with a correlation coefficient $(\mathrm{r})>0.31$. The average CLDQ score showed the strongest correlation with the domains of physical functioning $(\mathrm{r}=0.65)$, vitality $(\mathrm{r}=0.64)$, general health $(\mathrm{r}=0.625)$ and mental health $(\mathrm{r}=0.62)$, however it was more strongly associated with the PCS $(r=0.62)$ rather than $\operatorname{MCS}(\mathrm{r}=0.54)$.

\section{Structural Validity}

The exploratory factor analysis was carried out to assess the structural validity of the Hindi CLDQ. Table 5 shows the rescaled loading weights along with the Eigen values and the percentage of variance for the factors identified. The factor analysis explained 6 principal domains in Hindi CLDQ with an overall variability of $61.7 \%$. Majority of the items i.e. 24 out of $29(82.7 \%)$ items presented the highest loading weights. Of the 29, 12 items had a factor loading of $>0.7$ suggestive of a high variance contributors. The notable difference between the original English CLDQ and its Hindi translation was the inclusion of a new factor called 'sleep' which included questions related to sleep. Moreover some of the items of the domain of 'worry' of the original CLDQ were included into a larger factor called 'emotional function' in the Hindi version. The first domain (F1) included item numbers 10 (feeling anxious), 12 (felt unhappy), 18 (worry about the impact of liver disease on family), 19 (mood swings), 22 (worried about symptoms developing into major problem), 24 (feeling depressed), 25 (worried about condition getting worse), and 28 (worried about never feeling any better) and this domain was named as 'Emotional Function'. 
The second domain F2 comprised of item number 2 (felt tired or fatigued), 6 (problem of shortness of breath), 8 (bothered by decreased strength), 9 (trouble lifting or carrying heavy objects), 11 (felt a decreased level of energy) and this domain was called 'Fatigue'. The third factor included question number 16 (difficulty sleeping

Table 3: Comparison of CLDQ and SF36 domains between patients and healthy controls

\begin{tabular}{|c|c|c|c|c|c|c|c|}
\hline CLDQ domain & $\begin{array}{l}\text { Patients } \\
(n=494)\end{array}$ & $\begin{array}{l}\text { Controls } \\
(n=103)\end{array}$ & P value & $\begin{array}{l}\text { Child A } \\
(n=164)\end{array}$ & $\begin{array}{l}\text { Child B } \\
(n=198)\end{array}$ & $\begin{array}{l}\text { Child C } \\
(n=132)\end{array}$ & P value \\
\hline $\begin{array}{l}\text { Abdominal symptoms } \\
\text { (AS) }\end{array}$ & $4.91 \pm 1.64$ & $6.50 \pm 0.73$ & $0.0001 *$ & $5.41 \pm 1.50$ & $4.98 \pm 1.54$ & $4.23 \pm 1.66$ & $0.0001 *$ \\
\hline Fatigue $(\mathrm{F})$ & $4.24 \pm 1.54$ & $5.89 \pm 1.09$ & $0.0001 *$ & $4.71 \pm 1.50$ & $4.32 \pm 1.57$ & $3.59 \pm 1.32$ & $0.0001 *$ \\
\hline Systemic symptoms (SS) & $5.10 \pm 1.25$ & $6.22 \pm 0.94$ & $0.0001 *$ & $5.40 \pm 1.10$ & $5.18 \pm 1.25$ & $4.65 \pm 1.32$ & $0.0001 *$ \\
\hline Activity (A) & $4.66 \pm 1.86$ & $6.24 \pm 1.08$ & $0.0001 *$ & $5.35 \pm 1.50$ & $4.59 \pm 1.91$ & $3.93 \pm 1.91$ & $0.0001 *$ \\
\hline Emotional function (EF) & $4.72 \pm 1.40$ & $5.63 \pm 1.12$ & $0.0001 *$ & $5.19 \pm 1.30$ & $4.70 \pm 1.37$ & $4.21 \pm 1.37$ & $0.0001 *$ \\
\hline Worry $(\mathrm{W})$ & $4.70 \pm 1.52$ & $6.33 \pm 1.08$ & $0.0001 *$ & $5.17 \pm 1.41$ & $4.65 \pm 1.47$ & $4.26 \pm 1.54$ & $0.0001 *$ \\
\hline Average CLDQ score & $4.72 \pm 1.25$ & $6.14 \pm 0.84$ & $0.0001 *$ & $5.20 \pm 1.10$ & $4.73 \pm 1.24$ & $4.15 \pm 1.19$ & $0.0001^{*}$ \\
\hline \multicolumn{8}{|l|}{ SF 36 domains } \\
\hline Physical functioning (PF) & $54.4 \pm 31.5$ & $86.60 \pm 17.61$ & $0.0001 *$ & $66.2 \pm 27.7$ & $56.4 \pm 30.6$ & $38.1 \pm 30.4$ & $0.0001^{*}$ \\
\hline Role Physical (RP) & $40.8 \pm 35.37$ & $79.85 \pm 23.72$ & $0.0001 *$ & $50.7 \pm 35.4$ & $43.4 \pm 36.3$ & $24.4 \pm 28.0$ & $0.0001 *$ \\
\hline Body Pain (BP) & $59.06 \pm 30.26$ & $81.82 \pm 21.91$ & $0.0001 *$ & $64.7 \pm 29.0$ & $61.5 \pm 29.9$ & $49.6 \pm 30.0$ & $0.0001 *$ \\
\hline General Health (GH) & $45.84 \pm 24.15$ & $77.21 \pm 20.17$ & $0.0001 *$ & $52.2 \pm 26.0$ & $46.3 \pm 22.9$ & $37.5 \pm 20.2$ & $0.0001 *$ \\
\hline Vitality (VT) & $40.35 \pm 24.52$ & $71.23 \pm 19.24$ & $0.0001 *$ & $47.4 \pm 23.7$ & $41.2 \pm 25.0$ & $31.6 \pm 21.2$ & $0.0001 *$ \\
\hline Social Functioning (SF) & $75.17 \pm 30.42$ & $80.59 \pm 16.61$ & $0.0001 *$ & $78.2 \pm 27.5$ & $79.1 \pm 28.4$ & $65.9 \pm 34.5$ & $0.0002 *$ \\
\hline Role Emotional (RE) & $63.74 \pm 35.41$ & $77.99 \pm 25.05$ & $0.0001 *$ & $67.5 \pm 33.9$ & $65.0 \pm 34.7$ & $58.6 \pm 37.3$ & 0.099 \\
\hline Mental Health (MH) & $57.27 \pm 22.93$ & $72.47 \pm 19.93$ & $0.0001 *$ & $63.1 \pm 21.1$ & $56.8 \pm 24.1$ & $52.0 \pm 20.9$ & $0.0002 *$ \\
\hline $\begin{array}{l}\text { Physical component } \\
\text { summary (PCS) }\end{array}$ & $38.59 \pm 11.33$ & $53.71 \pm 7.06$ & $0.0001 *$ & $42.6 \pm 10.7$ & $39.6 \pm 10.7$ & $32.1 \pm 10.3$ & $0.0001 *$ \\
\hline $\begin{array}{l}\text { Mental component } \\
\text { summary (MCS) }\end{array}$ & $42.68 \pm 12.91$ & $48.76 \pm 9.04$ & $0.0001 *$ & $44.3 \pm 12.3$ & $42.9 \pm 12.9$ & $40.9 \pm 13.2$ & 0.0804 \\
\hline
\end{tabular}

Data expressed as mean $\pm S D$, * Significant at $p<0.05$

Table 4: Correlation between CLDQ and SF36 domains (r values)

\begin{tabular}{|c|c|c|c|c|c|c|c|c|c|c|}
\hline \multirow{2}{*}{ CLDQ domains } & \multicolumn{10}{|c|}{ SF-36 domains } \\
\hline & PF & $\mathbf{R} \mathbf{P}$ & $\mathbf{B P}$ & GH & VT & SF & $\mathbf{R E}$ & MH & PCS & MCS \\
\hline $\begin{array}{l}\text { Abdominal } \\
\text { symptoms }\end{array}$ & $0.4422 *$ & $0.3908^{*}$ & $0.5187 *$ & $0.4311^{*}$ & $0.4741^{*}$ & $0.3117 *$ & $0.3352 *$ & $0.4322 *$ & $0.4922 *$ & $0.3759 *$ \\
\hline Fatigue & $0.6090 *$ & $0.5156^{*}$ & $0.4722 *$ & $0.5159 *$ & $0.5995^{*}$ & $0.3542 *$ & $0.4205^{*}$ & $0.5149^{*}$ & $0.5921^{*}$ & $0.4505 *$ \\
\hline $\begin{array}{l}\text { Systemic } \\
\text { symptoms }\end{array}$ & $0.5298^{*}$ & $0.3559 *$ & $0.5297^{*}$ & $0.4525^{*}$ & $0.4593^{*}$ & $0.2921 *$ & $0.3776^{*}$ & $0.3954^{*}$ & $0.5270 *$ & $0.3554^{*}$ \\
\hline Activity & $0.6196^{*}$ & $0.4878^{*}$ & $0.3602 *$ & $0.4510 *$ & $0.5128 *$ & $0.3011 *$ & $0.3921^{*}$ & $0.4086^{*}$ & $0.5613^{*}$ & $0.3619 *$ \\
\hline Emotional function & $0.4863^{*}$ & $0.3886^{*}$ & $0.4581^{*}$ & $0.5609^{*}$ & $0.5458^{*}$ & $0.3921^{*}$ & $0.4710^{*}$ & $0.6410^{*}$ & $0.4350 *$ & $0.5847^{*}$ \\
\hline Worry & $0.4707^{*}$ & $0.3505^{*}$ & $0.4784^{*}$ & $0.6594 *$ & $0.5331^{*}$ & $0.3913^{*}$ & $0.4371^{*}$ & $0.6380^{*}$ & $0.4532 *$ & $0.5668^{*}$ \\
\hline Average CLDQ & $0.6493 *$ & $0.5158 *$ & $0.5714^{*}$ & $0.6255^{*}$ & $0.6405 *$ & $0.4167^{*}$ & $0.4968 *$ & $0.6176^{*}$ & $0.6291 *$ & $0.5485 *$ \\
\hline
\end{tabular}

*significant at $p<0.0001$ 
Table 5: Exploratory factors and explained variance after rotation for the Hindi CLDQ

\begin{tabular}{|c|c|c|c|c|c|c|}
\hline & \multicolumn{6}{|c|}{ Rotation sums of squared loadings } \\
\hline Factors & Item/Question & Rescaled loadings & Eigen Value & $\%$ of variance & $\begin{array}{l}\text { Cumulative } \\
\text { variance }\end{array}$ & $\begin{array}{l}\text { Cronbach's } \\
\text { alpha }\end{array}$ \\
\hline \multirow{8}{*}{$\begin{array}{l}\text { Factor } 1 \\
\text { 'Emotional } \\
\text { function' }\end{array}$} & Question 18 & 0.759 & \multirow{8}{*}{10.81} & \multirow{8}{*}{$37.27 \%$} & \multirow{8}{*}{37.27} & \multirow{8}{*}{.9022} \\
\hline & Question 22 & 0.751 & & & & \\
\hline & Question10 & 0.746 & & & & \\
\hline & Question24 & 0.730 & & & & \\
\hline & Question12 & 0.680 & & & & \\
\hline & Question 25 & 0.650 & & & & \\
\hline & Question 28 & 0.573 & & & & \\
\hline & Question 19 & 0.502 & & & & \\
\hline \multirow{5}{*}{$\begin{array}{l}\text { Factor } 2 \\
\text { 'Fatigue' }\end{array}$} & Question 9 & 0.720 & \multirow{5}{*}{1.96} & \multirow{5}{*}{$6.76 \%$} & \multirow{5}{*}{44.03} & \multirow{5}{*}{0.859} \\
\hline & Question11 & 0.715 & & & & \\
\hline & Question 8 & 0.707 & & & & \\
\hline & Question 2 & 0.617 & & & & \\
\hline & Question 6 & 0.604 & & & & \\
\hline \multirow{3}{*}{$\begin{array}{l}\text { Factor } 3 \\
\text { 'Sleep' }\end{array}$} & Question 16 & 0.740 & \multirow{3}{*}{1.65} & \multirow{3}{*}{$5.68 \%$} & \multirow{3}{*}{49.72} & \multirow{3}{*}{0.706} \\
\hline & Question 20 & 0.735 & & & & \\
\hline & Question 27 & 0.536 & & & & \\
\hline \multirow{4}{*}{$\begin{array}{l}\text { Factor } 4 \\
\text { 'Abdominal } \\
\text { Symptoms' }\end{array}$} & Question 14 & 0.675 & \multirow{4}{*}{1.33} & \multirow{4}{*}{$4.59 \%$} & \multirow{4}{*}{54.31} & \multirow{4}{*}{0.792} \\
\hline & Question 7 & 0.642 & & & & \\
\hline & Question 17 & \begin{tabular}{|l|}
0.582 \\
\end{tabular} & & & & \\
\hline & Question 1 & 0.540 & & & & \\
\hline \multirow{2}{*}{$\begin{array}{l}\text { Factor } 5 \\
\text { 'Activity' }\end{array}$} & Question 4 & 0.878 & \multirow{2}{*}{1.10} & \multirow{2}{*}{$3.81 \%$} & \multirow{2}{*}{58.12} & \multirow{2}{*}{0.896} \\
\hline & Question 13 & 0.846 & & & & \\
\hline \multirow{2}{*}{$\begin{array}{l}\text { Factor } 6 \\
\text { 'Pain' }\end{array}$} & Question 5 & 0.757 & \multirow{2}{*}{1.04} & \multirow{2}{*}{$3.58 \%$} & \multirow{2}{*}{61.70} & \multirow{2}{*}{0.613} \\
\hline & Question 3 & 0.670 & & & & \\
\hline
\end{tabular}

at night), 20 (unable to fall asleep at night), 27 (troubled by itching) and hence it was called 'Sleep'. The domain F4 composed of item numbers 1 (troubled by a feeling of abdominal bloating), 7 (not been able to eat as much as you would like), 14 (bothered by a limitation of your diet), 17 (troubled by a feeling of abdominal discomfort) and it was called 'Abdominal symptoms'. The fifth domain (F5) comprised of item numbers 4 (felt sleepy during the day), and 13 (felt drowsy), and was named as 'Activity'.The sixth domain (F6) comprised of question number 3 (experienced bodily pain) and 5 (experienced abdominal pain) and it was called 'Pain'. Factor number 1 owed maximum variability (37.27\%) in the Hindi CLDQ. The Cronbach's alpha of the 6 domains ranged from 0.61 (F6) to 0.90 (F1).
From the original 29 items, 5 items i.e. item number 15 (being irritable), 21 (having muscle cramps), 23 (having dry mouth), 26 (having problems concentrating) and 29 (concern about availability of liver for transplantation) did not get highlighted during the factor analysis as the factor loadings were $<0.5$ hence the analysis ignored them as redundant in comparison to the other factors for the Hindi CLDQ.

The mean CLDQ score ranged from 1 to 7 in patients and from 3.2 to 7 in controls. Most of the patients easily completed the questionnaire themselves however $20 \%$ required assistance from the interviewer. The completion rate of the data was $90 \%$ and only $10 \%$ reported any missing values thus indicating good acceptability of the Hindi CLDQ. The average time taken by the patients 
to fill the questionnaire was approximately 20 minutes. Most of the questions appeared to be clear and easily understandable. The Hindi CLDQ had good face validity.

\section{Diagnostic accuracy of Hindi CLDQ for assessing} HRQOL in patients with CLD

Using the average CLDQ score of healthy control as the gold standard we derived at a cut off of 6 with a sensitivity of 82.6 and specificity of 76.7. And subsequently the accuracy of this instrument in assessing the HRQOL in patients with CLD was described with the area under the ROC curve as 0.826 (Figure 1). Similarly the cut-off for the PCS and MCS of SF-36 were 49.1(AUC $=0.865$ ) and $47.8(\mathrm{AUC}=0.638)$ respectively.

\section{Discussion}

Chronic liver disease questionnaire (CLDQ) is the most widely used liver disease specific measure that specifically assesses the impact of liver disease on HRQOL. Based on the internationally accepted methodology for the translation and validation of HRQOL questionnaires we developed our Hindi version of CLDQ in patients with chronic liver disease. ${ }^{21}$

The Hindi version of CLDQ showed a good acceptability that could be administered with ease. It also showed high internal consistency with good reproducibility, and very good discriminant and convergent validity. The structural validity was comparable with the original English CLDQ, thus suggesting that the Hindi translation is semantically and functionally equivalent to the original English version. Using the average total score of CLDQ of the healthy control as gold standard the derived cut off 6 has a high sensitivity and specificity in discriminating the HRQOL in patients with CLD.

The Hindi version of CLDQ was also found to be very reliable. The homogeneity among the items, best assessed by Cronbach's alpha coefficient, in our study was 0.93 for the overall score. The maximum values for the same have reached 0.96 in Thai $^{13}$ version validation studies; our findings were comparable to those of the Greek $^{11}$, Spanish ${ }^{10}$ and Serbian ${ }^{22}$ studies.

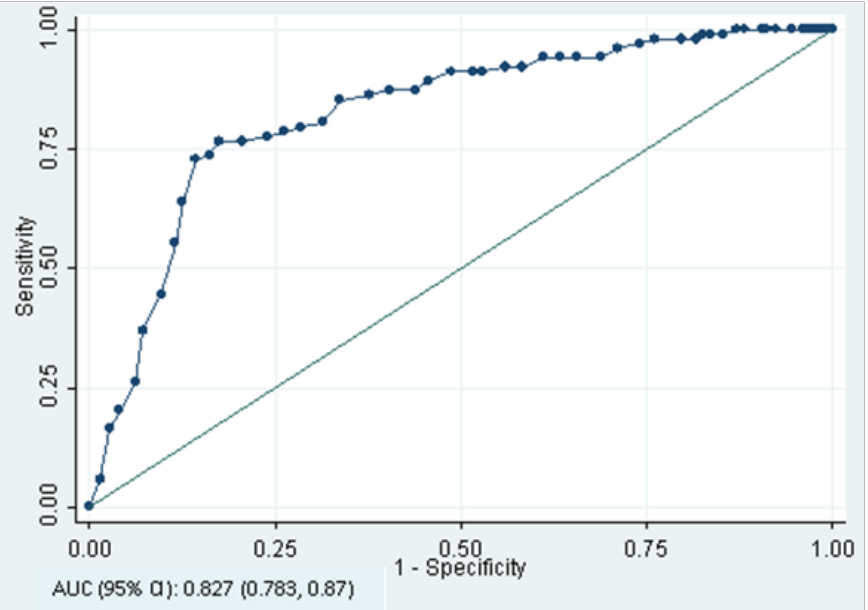

Figure 1: Receiver operating characteristic (ROC) curvefor average scoreof Hindi CLDQ(Cut off value $=6.0$ ).

The test retest reproducibility of Hindi version of CLDQ tested after a period of two weeks in a sub sample of 20 patients was very good with an ICC of 0.88 for the overall score. This was comparable to the Spanish ${ }^{10}$ study with the ICC of 0.90 in a subsample of 55 patients. Nonetheless in the original English study ICC of 0.59 was seen after repeating the test six months apart in 46 patients with CLD. Possibly a gap of six months was too long to expect clinical stability in such patients, which reflected in their low ICC.

The Hindi version of CLDQ exhibited good discriminant validity also. The overall CLDQ score as well as the domain sub scores showed an inverse relationship with the disease severity. Similar pattern of association between Child Pugh Score and CLDQ has been demonstrated by other studies also ${ }^{3,11,16,23-25}$. The post-hoc analysis revealed that the average and the sub scores of patients with Child $\mathrm{C}$ were significantly low than patients with both Child A and B, however most of the studies using CLDQ have not been able to differentiate between Child $\mathrm{C}$ and $\mathrm{B}^{3,6,26}$. Likewise the comparability of the scores of RE and MCS in SF-36 irrespective of the disease severity has been demonstrated by other studies also $0^{3,27,28}$. These findings suggest that a disease specific questionnaire like CLDQ is more sensitive in identifying the changes in subtle emotional issues also along with the clinical changes hence very well justifies the basic premise of using a disease specific questionnaire. 
Like the findings of the Spanish ${ }^{8}$ and Thai ${ }^{13}$ studies, the Hindi version of CLDQ also showed good convergent validity with a moderate to strong significant correlation between all the CLDQ domains and the SF-36 domains. Many of the other (Greek, ${ }^{11}$ Bengali, ${ }^{16}$ Serbian ${ }^{22}$ ) validation studies have not made any comparisons of CLDQ with any generic questionnaires, hence precluding comparisons. Unlike the Thai ${ }^{13}$ study where strong correlations were seen between the average CLDQ score and role emotional (RE) and mental health (MH) of SF-36 our study showed stronger and closer associations with the physical aspects like physical functioning (PF) general health $(\mathrm{GH})$ and vitality (VT) than the mental aspects like RE and mental component summary (MCS).

An exploratory factor analysis was carried out to test whether the Hindi translation of CLDQ has changed the structure of the original questionnaire or not. The Hindi version of CLDQ retained the original English version format of 6 domains, which explained an overall $61.6 \%$ of variability. This was close to the Greek ${ }^{11}$ version that explained the variability of $64.8 \%$ by 7 factors. This cumulative variance of our Hindi version of CLDQ was slightly lower than that explained by $\operatorname{Spanish}^{8}(68 \%)$ and Italian ${ }^{9}$ versions (65.4\%). There are also slight changes in the placement of items in the Hindi version. The total numbers of factors identified by the Italian ${ }^{9}$ study were five, while that of the Spanish ${ }^{8}$ and Greek ${ }^{11}$ were seven. The factor number 1 which we named as 'emotional function' retained all but one item number 29 (availability of liver) of the domain 'worry' of the original English CLDQ, but at the same time additionally included four items from the emotional function domain of the original questionnaire. Hence the factor number 1 is overall reflective of all the possible issues with the mental perturbations associated with chronic liver disease.

The factor number 2 identified as 'fatigue' included 3 items (number 11, 8, 2) from the fatigue domain of the original English questionnaire but incorporated item numbers 9 and 6 also under it. Thus, in the Hindi version the item no. 9 (trouble in lifting objects) and 6 (shortness of breath) originally under domain 'Activity' are suggestive of fatigue.

In this Hindi version, we identified a novel domain called 'sleep' which included 3 item numbers16, 20 and 27 and two of these questions were pertaining to the difficulty in sleep pattern such as item number 16 (inability to fall asleep) and item number 20 (difficulty in sleeping at night). Similar kind of new factor named as 'sleep' has been previously described by the Italian, ${ }^{9}$ Spanish, ${ }^{8}$ German, ${ }^{11}$ and Chinese ${ }^{15}$ studies also where the authors have discussed the cultural differences in the sleeping habits. Likewise, a Serbian ${ }^{22}$ study found a higher degree of belonging of item number 27 (Itching) to the domain of 'sleep' rather than 'systemic symptoms'. It is quite possible that the problem of itching could have been the cause of sleep disturbances in our patient population, thus it correlated well with the 'sleep' items.

Factor number 4 was identified as 'abdominal symptoms' not only included the items regarding the issues with abdominal discomfort (item no. 17) and bloating (item no.1) but also included the items related to food i.e. item number 7 (unable to eat) and 14 (troubled by the limitation of food), and, thus suggestive of a direct impact of the abdominal symptoms on the capacity to eat indicative of the dietary limitations. Interestingly, the Serbian ${ }^{22}$ version of CLDQ identifies the two item numbers 7 and 14 separately as an altogether new domain related to food termed as 'Nutrition'.

Of the 29 items, 5 items i.e. 15 (irritability), 21 (muscle cramps), number 23 (dry mouth), 26 (concentration) and 29 (availability of liver) were not pertinent to the Indian scenario as these items could not be categorized anywhere in any of the identified domains. Unlike the developed countries, liver transplantation is still an expensive treatment modality in India that is available only to a small fraction of patients, hence most patients do not relate to such questions. The Hindi translation highlights the fact that not just linguistic variations but also the related social, cultural and economic differences per se influence the basic structure of the health questionnaires.

Though CLDQ is a complete questionnaire covering most aspects of HRQOL however some items on the aspects of loneliness, sexual problems, joint pains, etc, may add to the overall meaning or information given by the CLDQ score. The major limitation of this study was that the responsiveness of CLDQ could not be assessed as done by other countries after liver transplantation. 
India is a diverse nation, often called the "tower of Babel" where the common parlance of Hindi is practically influenced by Persian, Urdu, English and the local dialects. Profound linguistic diversity even in the neighboring regions and states influences the understanding and perception of the items and subsequently their placements under various domains. Hence this variability underlines the necessity for cultural adaptations and validations before being used. Nevertheless, the Hindi translation of CLDQ has been well accepted with good face validity in the present study.

\section{Acknowledgement}

We thank Dr. Zobair M. Younossi for giving us the permission to translate the original English CLDQ to Hindi language.

The questionnaire is available on

\section{www.tropicalgastro.com}

\section{References}

1. Saab S, Bownik H, Ayoub N et al. Differences in healthrelated quality of life scores after orthotopic liver transplantation with respect to selected socioeconomic factors. Liver Transpl. 2011;17(5):580-90.

2. Martin LM1, Sheridan MJ, Younossi ZM. The impact of liver disease on health-related quality of life: a review of the literature. Curr Gastroenterol Rep. 2002;4(1):79-83.

3. Younossi ZM, Guyatt G, Kiwi M et al. Development of a disease specific questionnaire to measure health related quality of life in patients with chronic liver disease. Gut. 1999;45(2):295-300.

4. Bayliss MS. Methods in outcomes research in hepatology: definitions and domains of quality of life. Hepatology. 1999;29(6 Suppl):3S-6S.

5. Younossi ZM, Kiwi ML, Boparai Net al.Cholestatic liver diseases and health-related quality of life. $\mathrm{Am} \mathrm{J}$ Gastroenterol. 2000;95(2):497-502.

6. Younossi ZM, Boparai N, Price LLet al. Health-related quality of life in chronic liver disease: the impact of type and severity of disease. Am J Gastroenterol. 2001;96(7):2199205.

7. Häuser W, Schnur M, Steder-Neukamm Uet al. Validation of the German version of the Chronic Liver
Disease Questionnaire. Eur J Gastroenterol Hepatol. 2004;16(6):599-606.

8. Ferrer M, Córdoba J, Garin O et al.Validity of the Spanish version of the Chronic Liver Disease Questionnaire (CLDQ) as a standard outcome for quality of life assessment. Liver Transpl. 2006;12(1):95-104.

9. Rucci P, Taliani G, Cirrincione L et al. Validity and reliability of the Italian version of the Chronic Liver Disease Questionnaire (CLDQ-I) for the assessment of healthrelated quality of life. Dig Liver Dis. 2005;37(11):850-60.

10. Benito de Vale M, Josefsson A, Lindkvist B et al. Validation of the Swedish version of the chronic liver disease questionnaire. Scand J Gastroenterol. 2012;47(5):614-5.

11. Kollia Z, Patelarou E, Vivilaki Vet al. Translation and validation of the Greek chronic liver disease questionnaire. World J Gastroenterol. 2010;16(46):5838-44.

12. Mahmoudi H, Jafari P, Alizadeh-NainiMcetal.Validity and reliability of Persian version of Chronic Liver Disease Questionnaire (CLDQ). Qual Life Res. 2012;21(8):147985.

13. Sobhonslidsuk A, Silpakit C, Kongsakon R et al. Chronic liver disease questionnaire: translation and validation in Thais. World J Gastroenterol. 2004;10(13):1954-7.1.

14. Tanaka A, Kikuchi K, Miura R et al. Validation of the Japanese version of the Chronic Liver Disease Questionnaire for the assessment of health-related quality of life in patients with chronic viral hepatitis. Hepatol Res. 2016;46(3):E45-50.

15. Lam ET, Lam CL, Lai CL et al. Psychometrics of the chronic liver disease questionnaire for Southern Chinese patients withchronic hepatitis B virus infection. World $J$ Gastroenterol. 2009;15(26):3288-97.

16. Ray I, Dutta D, Basu P et al. Quality of life assessment of patients with chronic liver disease in eastern India using a Bengali translation chronic liver disease questionnaire. Indian J Gastroenterol. 2010;29(5):187-95.

17. Ranawaka CK, Miththinda JK, Senanayake SM et al.Validation of the Sinhala version of the Chronic Liver Disease Questionnaire (CLDQ) for assessment of health related quality of life among Sri Lankan cirrhotics. Ceylon Med J. 2013;58(4):156-62.

18. Ware JE Jr, Sherbourne CD. The MOS 36-item short-form health survey (SF-36). I. Conceptual framework and item selection. Med Care. 1992;30(6):473-83.

19. Ware JE, Jr.Kosinski MK, Bjorner JB et al. User's Manual For SF-36v2 Health Survey. (2nd Ed.). Lincoln, RI: Quality Metric Incorporated 2008.

20. Guillemin F, Bombardier C, Beaton D. Cross-cultural 
adaptation of health-related quality of life measures: literature review and proposed guidelines. J ClinEpidemiol. 1993;46(12):1417-32.

21. Beaton DE, Bombardier C, Guillemin F et al. Guidelines for the process of cross-cultural adaptation of self-report measures. Spine (Phila Pa 1976). 2000;25(24):3186-91.

22. Popovic DD, Kovacevic NV, KisicTepavcevic DB et al. Validation of the chronic liver disease questionnaire in Serbian patients. World $J$ Gastroenterol. 2013;19(30):4950-7.

23. Sumskiene J, Sumskas L, Petrauskas D et al. Diseasespecific health-related quality of life and its determinants in liver cirrhosis patients in Lithuania. World J Gastroenterol. 2006;12(48):7792-7.

24. Les I, Doval E, Flavià M et al.Quality of life in cirrhosis is related to potentially treatable factors. Eur J Gastroenterol Hepatol. 2010;22(2):221-7.
25. Zuberi BF1, Memon AR, Afsar S et al. Correlation of quality of life in patients of cirrhosis of liver with etiology and disease severity using disease-specific quality of life questionnaire. J Ayub Med CollAbbottabad. 2007;19(2): 7-11.

26. Bao ZJ, Qiu DK, Ma X et al. Assessment of health-related quality of life in Chinese patients with minimal hepatic encephalopathy. World J Gastroenterol. 2007;13(21): 3003-8.

27. Arguedas MR, DeLawrence TG, McGuire BM.Influence of hepatic encephalopathy on health-related quality of life in patients with cirrhosis. Dig Dis Sci. 2003;48(8):1622-6.

28. Kanwal F, Hays RD, Kilbourne AMet al.Are physicianderived disease severity indices associated with healthrelated quality of life in patients with end-stage liver disease? Am J Gastroenterol. 2004;99(9):1726-32. 